Abanico Agroforestal. Enero-Diciembre 2021; 3:1-10. http://dx.doi.org/10.37114/abaagrof/2021.3 Artículo Original. Recibido: 04/01/2020. Aceptado: 22/03/2021. Publicado: 12/04/2021. Clave:2020-20.

\title{
Variables hematológicas en aves deportivas, ganso común, pato doméstico, pato azteca, guajolote y pollo de engorda
}

\author{
Hematological variables in sport birds, common goose, domestic duck, \\ Aztec duck, turkey and broiler chicken
}

\section{Sánchez-Torres Laura*1 ID, Arredondo-Castro Mauricio ${ }^{2 I D}$, Orozco-Benítez Guadalupe ${ }^{3 \mathrm{ID}}$, Gutiérrez-Arenas Diana2 ID, Carrillo-Beltrán Julio ${ }^{4 \mathrm{ID}}$, Lepe-Aguilar Rosa ${ }^{3 \mathrm{ID}}$, Avila- Ramos Fidel ${ }^{\star \star 2}$ ID}

\begin{abstract}
${ }^{1}$ Maestría en Producción Pecuaria; Universidad de Guanajuato, Campus Irapuato-Salamanca, División Ciencias de la Vida. ${ }^{2}$ División Ciencias de la Vida, Universidad de Guanajuato, Programa Educativo de Medicina Veterinaria y Zootecnia. México. ${ }^{3}$ Unidad Académica de Medicina Veterinaria y Zootecnia de la Universidad Autónoma de Nayarit; Nayarit, México. ${ }^{4}$ Unidad Académica de Contaduría y Administración de la Universidad Autónoma de Nayarit; Nayarit, México *Autor responsable: Laura Selena Sánchez-Torres. ${ }^{* *}$ Autor de correspondencia: Fidel Avila Ramos, Programa Educativo de Medicina Veterinaria y Zootecnia, Ex Hacienda El Copal km. 9; carretera Irapuato-Silao; A.P. 311; C.P. 36500; Irapuato, Guanajuato. México. E-mail: sanchez.torres122@outlook.com, arredondo.m@ugto.mx, mgorozco63@gmail.com, diana.gutierrez@ugto.mx, doctorjuliocarrillo@gmail.com,
\end{abstract} isela.aguilar@uan.edu.mx, ledifar@ugto.mx.

\section{RESUMEN}

El análisis sanguíneo en aves es una evidencia que permite evaluar su estado de salud y es una herramienta diagnóstica para el médico veterinario clínico. El objetivo de la investigación fue conocer las variables hematológicas de aves comunes, deportivas, ganso común, pato doméstico, pato azteca, guajolote y pollo de engorda por sexo, especie y la diferencia entre las especies. Se tomó una muestra de sangre a 88 aves por venopunción de la vena ulnar usando ácido etilendiaminotetraacético (EDTA) como anticoagulante. Se contaron los eritrocitos y los leucocitos totales por el método de Natt y Herriks. El diferencial de leucocitos se realizó por medio de un frotis sanguíneo, se midió la concentración de hemoglobina con la técnica de cianometahemoglobina y el porcentaje de microhematocrito. Los datos fueron comparados con un análisis de varianza en un diseño completamente al azar y las diferencias se compararon con la prueba de Tukey $(P<0.05)$. Para eosinófilos y basófilos se utilizó la prueba de Kruskal-Wallis debido a que los datos no presentaron normalidad. Las variables hematológicas en aves deportivas, ganso común, pato doméstico, pato azteca, guajolote y pollo de engorda fueron similares para hembras y machos de la misma especie. Pero hubo diferencias en células blancas de las especies estudiadas.

Palabras clave: aves domésticas, hematología, Natt y Herriks.

\begin{abstract}
Blood analysis in birds is an evidence that allows evaluating their health status and is a diagnostic tool for the clinical veterinarian. The objective of the research was to know the hematological variables of common birds, sport birds, common goose, domestic duck, Aztec duck, turkey and broiler chicken by sex, species and the difference between species. A blood sample was taken from 88 birds by venipuncture of the ulnar vein using EDTA as anticoagulant. Erythrocytes and total leukocytes were counted by the method of Natt and Herriks. Leukocyte differential was performed by blood smear, hemoglobin concentration was measured by the cyanomethemoglobin technique and microhematocrit percentage. Data were compared with an analysis of variance in a completely randomized design and differences were compared with Tukey's test $(\mathrm{P}<0.05)$. For eosinophils and basophils, the Kruskal-Wallis test was used because the data did not present normality. The hematological variables in sport birds, common goose, domestic duck, Aztec duck, turkey and broiler chicken were similar for females and males of the same species. But there were differences in white cells of the species studied.
\end{abstract}

Keywords: domestic fowl, hematology, Natt and Herriks. 


\section{INTRODUCCIÓN}

En México, las aves deportivas, el ganso común, el pato doméstico, el pato azteca, el guajolote y el pollo de engorda son encontrados en granjas comerciales, pequeñas explotaciones de traspatio, reservas animales o incluso son adoptados como animales de compañía (CucaGarcía et al., 2015; Pineda-Leyva et al., 2015). El conocimiento de sus parámetros hematológicos es una herramienta general para conocer su estado de salud con usos básicos en la clínica de aves e investigación científica (Mitchell y Johns, 2008).

La sangre es un tejido que consta de una parte liquida y de componentes celulares; se encarga principalmente de captar, transportar y distribuir nutrientes en el organismo a través de los vasos sanguíneos. Su evaluación se realiza a través de un hemograma donde se expresa la población de eritrocitos y leucocitos, además, permite identificar alteraciones morfológicas y valorar su funcionamiento (Campbell, 2013; Montalvo, 2017).

En las aves y los reptiles el hemograma se realiza manualmente debido a sus eritrocitos y trombocitos nucleados. Además, los leucocitos presentan múltiples formas y el tamaño celular es distinto en comparación a los mamíferos, por lo que el conteo total de células rojas (CCR) y blancas (CCB) es directo a través de un hematocitómetro (Martínez-Silvestre et al., 2011). Para facilitar su conteo se utiliza la solución de Natt y Herriks que tiñe a las células mejorando su contraste, para leucocitos se obtiene un resultado general y para conocer la población especifica se realiza un frotis sanguíneo (Campbell, 2013). Para identificar heterófilos (Het), eosinófilos (Eos), basófilos (Bas), linfocitos (Linf), monocitos (Mon) y trombocitos (Trom) (Martinho, 2009).

De manera indirecta evaluamos la serie roja midiendo el porcentaje de hematocrito (Hto) que demuestra la proporción de glóbulos rojos presentes en la sangre, además reporta alteraciones en el suero como hemolisis, ictericia o lipemia, un hematocrito bajo puede sugerir anemia, deshidratación o policitemia (Agustí, 2015). La hemoglobina $(\mathrm{Hb})$ es una proteína contenida en los eritrocitos y da el color rojo a la sangre, sirve para transportar el $0_{2}$ y si la cantidad es reducida indica (hipocrómico), si es normal (normocrómico) y por lo tanto su funcionamiento (Gálvez et al., 2009). En aves la heterofilia y linfopenia son indicadores de estrés, inmunosupresión e infecciones activas (Garbus et al., 2019). Sin embargo, en las aves las variables hematológicas pueden modificarse por diferentes factores como es la edad, raza de ave, locación de las especies y su comportamiento migratorio (Pistone et al., 2017; Bílcová et al., 2017). Por lo tanto, es necesario conocer los parámetros hematológicos por zona geográfica y el objetivo de la investigación fue conocer las variables hematológicas de las especies más comunes de aves domésticas para tener valores de referencia clínica.

\section{MATERIAL Y MÉTODOS}

\section{Ubicación del estudio}

Las muestras sanguíneas se colectaron de aves obtenidas en el municipio de Irapuato, Guanajuato, México, localizado a 1,715 msnm, con clima cálido subhúmedo y precipitaciones en verano (INEGI, 2017). Las muestras se analizaron en el laboratorio de Avicultura de la Universidad de Guanajuato, en la División Ciencias de la Vida, Campus Irapuato- Salamanca, km 9 carretera Irapuato-Silao en Irapuato, Guanajuato. 


\section{Toma de muestras sanguíneas}

Se realizó venopunción de la vena ulnar en todas las aves, haciendo previa asepsia del área con torundas impregnadas con alcohol, se utilizaron jeringas de $3 \mathrm{~mL}$ y agujas calibre 23G, se colecto $1 \mathrm{~mL}$ de sangre en tubos vacutainer ${ }^{\circledR}$ con EDTA.

\section{Aves}

Se realizó un muestreo por conveniencia de 88 aves adultas, de las cuales 20 aves fueron deportivas (Gallus gallus) 10 hembras y 10 machos, 20 Guajolotes (Meleagris gallopavo) 10 machos y 10 hembras, 8 patos azteca (Cairina moschata) 5 machos y 4 hembras, 20 patos domésticos (Anas platyrhynchos) 10 machos y 10 hembras, 20 gansos (Anser anser) 10 hembras y 10 machos, 20 pollos de engorda (Gallus gallus domesticus), 10 hembras y 10 machos.

\section{Frotis sanguíneo}

Para el extendido se utilizaron portaobjetos comerciales deslizando la sangre sobre el portaobjetos y secado al aire para teñir posteriormente con la tinción Dip Quick stain ${ }^{\circledR}$.

\section{Método para contar células sanguíneas}

El conteo de células sanguíneas se realizó con ayuda de una cámara de Neubauer (Marienfeld, Alemania) usando la solución Natt y Herriks. Para obtener la cantidad total de células se utilizaron las siguientes fórmulas:

CCR $(\mathrm{mm} 3)=H$ (hematíes contados) $\times 5 \times 10 \times D$ (factor de dilución 1:200). CCB $(\mathrm{mm} 3)=L$ (leucocitos contados) $/ 4 \times 10 \times D$ (factor de dilución 1:20).

\section{Diferencial de leucocitos}

Para realizar el diferencial de leucocitos se contaron 100 células, observando con el objetivo 100 x con aceite de inmersión, siguiendo un patrón de observación en forma de zigzag, para obtener resultados más precisos de la población de células blancas.

\section{Hematocrito}

El capilar se colocó en la centrífuga (TG12M, microhematocrit centrifuge) de manera horizontal, dejando el extremo sellado hacia afuera, se programó a 2500 rpm durante 10 min y se utilizó la siguiente fórmula para obtener el porcentaje: Hto $(\%)=L 2$ (hematíes en $\mathrm{mm}$ ) / L1 (hematíes + plasma en $\mathrm{mm}){ }^{*} 100$ (Campbell, 2013).

\section{Hemoglobina}

Se colocaron $5 \mathrm{~mL}$ de reactivo Drabkin (Hycel) (cianometahemoglobina) y $20 \mu \mathrm{l}$ de sangre con anticoagulante en un frasco ámbar, se mezcló de manera gentil para homogeneizarlas, se dejó reposar durante 5 minutos y se midió la absorbancia a 540 nm en un espectrofotómetro (Epoch, Biotech), el resultado fue multiplicado por 36.77 para obtener la concentración de hemoglobina en g/dL (Samour, 2007). 


\section{Análisis estadístico}

Se realizó un análisis de varianza con un diseño completamente al azar $(\mathrm{P}<0.05)$, donde se consideraron como variables dependientes el conteo de eritrocitos, leucocitos, heterófilos, linfocitos, monocitos, trombocitos, hematocrito y hemoglobina en las aves y como variables independientes el sexo y la especie. Para eosinófilos y basófilos se utilizó la prueba de KruskalWallis debido que los datos no presentaron normalidad. Posteriormente, se realizó una comparación múltiple de medias con el método Tukey $(\mathrm{P}<0.05)$ con el programa estadístico statgraphics centurión XV.

\section{RESULTADOS}

Las variables hematológicas en aves deportivas, ganso común, pato doméstico, pato azteca, guajolote y pollo de engorda fueron similares para hembras y machos de la misma especie (Cuadro 1).

\section{Hembras}

En el Cuadro 1 el número de eritrocitos totales del ganso común fue menor al compararlo con el del pato doméstico, pato azteca y guajolote $(\mathrm{P}<0.05)$; así mismo, el conteo de leucocitos en las aves deportivas fue mayor $(\mathrm{P}<0.05)$ que en el ganso común y pato doméstico. En el diferencial de leucocitos, la cantidad de heterófilos fue similar entre las seis especies. Por otro lado, los eosinófilos presentaron mayor diferencia $(P<0.05)$ entre el ganso común y el guajolote. El porcentaje de basófilos resulto mayor en el pato doméstico $(P<0.05)$ en relación a las aves deportivas y pollo de engorda. El índice de linfocitos en aves deportivas y pollo de engorda sobresalió de lo registrado para patos domésticos, patos azteca y guajolote $(P<0.05)$. El número de monocitos en el guajolote fue más que el observado en el pollo de engorda, ave deportiva y ganso común $(\mathrm{P}<0.05)$. Además, se observó el hematocrito más alto en el pato azteca $(P<0.05)$ y el más bajo en el pollo de engorda, guajolote y ganso común. La presencia de trombocitos aumentó en el ganso común y pato azteca $(\mathrm{P}<0.05)$ comparado con las aves deportivas, la concentración de hemoglobina fue mayor en el pato azteca $(P<0.05)$.

\section{Machos}

En el Cuadro 1 el conteo de células rojas en aves deportivas y pato doméstico fue mayor $(P<0.05)$ que lo observado en ganso común y pollo de engorda, pero en células blancas el pato doméstico y guajolote son mayores $(\mathrm{P}<0.05)$ comparados con el ganso común y ave deportiva. Por otro lado, en el diferencial de leucocitos los valores de heterófilos, basófilos y monocitos fueron similares para las seis especies estudiadas. Los eosinófilos fueron más frecuentes en el pato doméstico $(P<0.05)$ comparados con el guajolote. La cantidad de linfocitos es mayor en aves deportivas $(\mathrm{P}<0.05)$ en relación al ganso común, pato doméstico, pato azteca y pollo de engorda. El porcentaje de hematocrito resultó más elevado en aves deportivas $(P<0.05)$ comparadas con el ganso común, pato doméstico, guajolote y pollo de engorda. Los trombocitos se presentaron en mayor medida en ganso común, pato doméstico y pato azteca $(\mathrm{P}<0.05)$, seguidos por las aves deportivas y guajolotes, la menor cantidad $(P<0.05)$ la presentó el pollo de engorda. La concentración de hemoglobina destacó en el pato azteca $(\mathrm{P}<0.05)$ con respecto a las demás especies. 
Cuadro 1. Parámetros hematológicos en hembras y machos de aves deportivas, ganso común, pato doméstico, pato azteca, guajolote y pollo de engorda.

\begin{tabular}{|c|c|c|c|c|c|c|c|}
\hline $\begin{array}{c}\text { Parámetro } \\
\%\end{array}$ & Ave deportiva & Ganso común & Pato doméstico & Pato azteca & Guajolote & Pollo de engorda & EE \\
\hline \multicolumn{8}{|c|}{ Hembras } \\
\hline $\mathrm{CCR} \mathrm{mm}^{3}$ & $337.3 \pm 49.1 \mathrm{bc}$ & $313.0 \pm 55.6^{c}$ & $437.9 \pm 77.8^{a}$ & $461.0 \pm 83.9^{a}$ & $417.8 \pm 83.9 \mathrm{ab}$ & $361.7 \pm 38.9 \mathrm{abc}$ & 20.6 \\
\hline $\mathrm{CCB} \mathrm{mm}^{3}$ & $565.9 \pm 176.9 a$ & $271.5 \pm 92.2^{c}$ & $356.2 \pm 147.7 b c$ & $405.2 \pm 72.9 \mathrm{abc}$ & $442.2 \pm 187.2 \mathrm{abc}$ & $540.5 \pm 145.1 \mathrm{ab}$ & 47.9 \\
\hline Het & $19.4 \pm 7.6^{a}$ & $27.8 \pm 11.1^{a}$ & $27.6 \pm 8.5^{a}$ & $31.7 \pm 4.5^{a}$ & $25.7 \pm 5.9$ & $22.4 \pm 5.3^{a}$ & 2.4 \\
\hline Eos & $1.2 \pm 1.6 \mathrm{ab}$ & $3.3 \pm 2.6^{a}$ & $2.9 \pm 3.3^{a b}$ & $1.0 \pm 1.1 \mathrm{ab}$ & $0.2 \pm 0.6^{b}$ & $1.5 \pm 1.9 a b$ & 0.6 \\
\hline Bas & $0.0 \pm 0.0^{b}$ & $0.2 \pm 0.7 \mathrm{ab}$ & $1.0 \pm 1.1^{\mathrm{a}}$ & $0.0 \pm 0.0 \mathrm{ab}$ & $0.3 \pm 0.9 a b$ & $0.0 \pm 0.0^{b}$ & 0.2 \\
\hline $\operatorname{Lin}$ & $40.0 \pm 11.5^{a}$ & $28.3 \pm 15.1 \mathrm{ab}$ & $19.4 \pm 6.9^{b}$ & $21.0 \pm 5.9^{b}$ & $21.4 \pm 10.8^{b}$ & $39.5 \pm 8.4^{a}$ & 3.3 \\
\hline Mon & $39.1 \pm 9.5 \mathrm{bc}$ & $40.2 \pm 7.0 \mathrm{bc}$ & $49.2 \pm 6.3 \mathrm{ab}$ & $46.2 \pm 7.8^{a b c}$ & $52.4 \pm 7.8^{a}$ & $36.6 \pm 8.2^{c}$ & 2.5 \\
\hline Hto & $46.6 \pm 5.7 \mathrm{ab}$ & $41.8 \pm 3.9 \mathrm{bc}$ & $47.4 \pm 4.4^{\mathrm{ab}}$ & $54.6 \pm 2.0^{a}$ & $45.1 \pm 4.0 \mathrm{bc}$ & $39.4 \pm 4.1^{c}$ & 1.4 \\
\hline Tromb $\mu \mathrm{L}$ & $33.3 \pm 5.9^{b}$ & $43.2 \pm 6.7^{a}$ & $39.1 \pm 6.7 \mathrm{ab}$ & $45.0 \pm 7.6^{a}$ & $39.8 \pm 5.5^{a b}$ & $40.7 \pm 4.3 \mathrm{ab}$ & 1.9 \\
\hline $\mathrm{Hb} g / \mathrm{dL}$ & $11.9 \pm 2.9^{c}$ & $16.8 \pm 5.5^{b}$ & $15.0 \pm 2.2 \mathrm{bc}$ & $32.4 \pm 2.6^{a}$ & $12.1 \pm 0.9^{c}$ & $14.0 \pm 1.8 \mathrm{bc}$ & 0.9 \\
\hline \multicolumn{8}{|c|}{ Machos } \\
\hline $\mathrm{CCR} \mathrm{mm}^{3}$ & $440.9 \pm 89.8^{a}$ & $318.5 \pm 54.9 \mathrm{~cd}$ & $426.7 \pm 96.4^{a}$ & $424.2 \pm 73.0^{a b}$ & $358.5 \pm 55.6 \mathrm{ab}$ & $257.2 \pm 48.3^{d}$ & 22.7 \\
\hline $\mathrm{CCB} \mathrm{mm}^{3}$ & $234.8 \pm 63.0 \mathrm{bc}$ & $221.7 \pm 57.5^{c}$ & $424.4 \pm 96.8^{a}$ & $335.2 \pm 117.0 \mathrm{abc}$ & $434.6 \pm 120.1^{a}$ & $379.6 \pm 167.4^{\mathrm{ab}}$ & 34.6 \\
\hline Het & $22.6 \pm 9.4^{\mathrm{a}}$ & $21.3 \pm 8.3^{a}$ & $28.9 \pm 9.1^{\mathrm{a}}$ & $29.8 \pm 3.1^{a}$ & $23.9 \pm 10.8^{a}$ & $23.6 \pm 8.8^{a}$ & 2.8 \\
\hline Eos & $0.4 \pm 0.8 \mathrm{ab}$ & $2.3 \pm 2.9 \mathrm{ab}$ & $2.5 \pm 2.4^{\mathrm{a}}$ & $0.8 \pm 1.0 \mathrm{ab}$ & $0.0 \pm 0.0^{b}$ & $0.3 \pm 0.6 \mathrm{ab}$ & 0.5 \\
\hline Bas & $0.0 \pm 0.0^{a}$ & $0.0 \pm 0.0^{a}$ & $1.2 \pm 2.2^{a}$ & $0.0 \pm 0.0^{a}$ & $0.0 \pm 0.0^{a}$ & $0.0 \pm 0.0^{a}$ & 0.2 \\
\hline Lin & $37.9 \pm 12.1^{a}$ & $27.1 \pm 17.2^{b}$ & $18.9 \pm 8.4^{b}$ & $22.4 \pm 7.1^{b}$ & $28.3 \pm 12.5 \mathrm{ab}$ & $21.6 \pm 6.3^{b}$ & 3.6 \\
\hline Mon & $38.9 \pm 11.3^{a}$ & $48.9 \pm 16.1^{a}$ & $48.6 \pm 10.1^{a}$ & $47.0 \pm 6.0^{a}$ & $47.9 \pm 11.2^{a}$ & $54.4 \pm 12.2^{a}$ & 3.8 \\
\hline Hto & $54.1 \pm 4.9^{a}$ & $40.4 \pm 3.4^{\mathrm{cd}}$ & $45.0 \pm 3.3 \mathrm{bc}$ & $51.2 \pm 4.3 \mathrm{ab}$ & $46.4 \pm 4.9^{b}$ & $34.6 \pm 5.4 \mathrm{~d}$ & 1.4 \\
\hline Tromb $\mu \mathrm{L}$ & $34.5 \pm 4.8^{b}$ & $41.5 \pm 3.6^{a}$ & $42.4 \pm 7.1^{a}$ & $42.6 \pm 1.8^{a}$ & $33.8 \pm 5.0^{b}$ & $19.9 \pm 2.1^{c}$ & 1.4 \\
\hline $\mathrm{Hb} g / \mathrm{dL}$ & $16.1 \pm 2.9^{b}$ & $17.3 \pm 6.0^{b}$ & $14.3 \pm 1.8^{b}$ & $33.5 \pm 4.1^{a}$ & $14.0 \pm 2.29 b$ & $14.4 \pm 1.2^{b}$ & 1.0 \\
\hline
\end{tabular}

a-d Literales distintas por fila refieren diferencia estadística significativa $(\mathrm{P}<0.05)$.

CCR $\mathrm{mm}^{3}=$ Eritrocitos totales por $\mathrm{mm}^{3}$, CCB $\mathrm{mm}^{3}=$ Leucocitos totales por $\mathrm{mm}^{3}$, Het=heterófilos, Eos=Eosinófilos, Bas=Basófilos, Lin=Linfocitos, Mon=Monocitos, $\mathrm{H}$ to=Hematocrito, Tromb $\mu \mathrm{L}=$ Trombocitos por $\mu \mathrm{L}, \mathrm{Hb} g / \mathrm{dL}=$ Hemoglobina en $\mathrm{g}$ por $\mathrm{dL}$. 


\section{DISCUSIÓN}

Las variables hematológicas son elementos básicos para conocer y evaluar la salud general de las aves, saber los parámetros por especie y las diferencias que tienen las hembras comparadas con los machos permite identificar desequilibrios en sus poblaciones celulares (Fairbrother y O'Loughlin, 1990; Mitchell y Johns, 2008). Okeudo et al. (2003), Aengwanich y Tanomtong (2007), Azeez et al. (2011), Albokhadaim (2012), Lashev et al. (2015) reportan diferencia en eritrocitos totales, hemoglobina y hematocrito relacionado al sexo del ave. En machos el índice aumenta y en hembras disminuye debido al nivel de testosterona que tiene efecto directo sobre la eritropoyesis (Gonzales, 2011; Campbell, 2015). Fairbrother y O'Loughlin (1990), Moreira et al. (2009) y en el presente estudio el efecto hormonal no fue significativo, pero mostro tendencias similares. Es posible que haya especies de aves que puedan manifestar de esa forma y otras no presenten el mismo efecto como se presenta el dimorfismo sexual en aves.

\section{Conteo de eritrocitos totales}

Los eritrocitos transportan y distribuyen el oxígeno y dióxido de carbono en el cuerpo (Scanes, 2015). Okeudo et al. (2013) reportan menos CCR comparado a nuestro estudio en hembras y machos del pato azteca. Campbell (2015) menciona que los patos tienen valores más altos de CCR comparados con los gansos debido al fotoperiodo. Pineda-Leiva et al. (2015) obtuvieron valores menores en machos de combate. Pero Moreira et al. (2009) describen valores mayores a los observados en nuestro estudio para hembras y machos de guajolote. La cantidad de eritrocitos puede asociarse al tipo de alimentación en las especies domésticas (Lashev et al., 2015). Además, se puede presentar variación entre las especies debido a la temporada de cría y la relación entre su peso y tasa metabólica (McKechnie, 2007).

\section{Conteo de leucocitos totales}

Los leucocitos defienden al cuerpo de los patógenos o los agentes extraños (Galvez et al., 2009). Lashev et al. (2015) observaron variación de ésta línea celular relacionada a las vacunas, la dieta o las condiciones higiénicas en el galpón de las aves. Pineda-Leyva et al. (2015) obtuvieron menor CCB en machos de combate. En nuestros resultados tanto hembras como machos de pollo de engorda superaron el promedio observado por Scanes (2015). Por otro lado, Moreira et al. (2009) señalan un CCB mayor en hembras y machos de guajolote.

Azeez et al. (2011) han descrito altos conteos de CCB en aves jóvenes como es para pollo de engorda en este estudio indicando las diferencias con las otras especies puede deberse a la edad y hematopoyesis no madura. Foo et al. (2017) indican que los caracteres sexuales secundarios en las especies son desarrollados por el nivel de testosterona que en aves tiene efecto inmunomodulador. Por lo tanto, la diferencia entre las especies puede ser a causa por el desarrollo del plumaje, su color e incluso el grado de agresividad que pueden manifestar.

\section{Diferencial de leucocitos}

Es un estudio que permite distinguir los tipos de leucocitos para interpretar de manera más precisa el hemograma (Galvez et al., 2009). Lashev et al. (2015) no observaron diferencias entre codornices, aves de combate, pollo de engorda, guajolote y faisán. Los parámetros observados en el presente estudio fueron menores a los promedios reportados por Scanes (2015), excepto, en los monocitos que se presentan en cantidades mayores. Okeudo et al. (2003) reportan menos heterófilos en hembra y macho en pato azteca, eosinófilos más altos y linfocitos en mayor cantidad comparado a los nuestros. Oloyemi y Arewolo (2009) reportan en 
pato doméstico parámetros mayores de linfocitos, heterófilos y menores de monocitos y eosinófilos. Los heterófilos y los linfocitos son leucocitos predominantes asociados a estresores, infecciones, inflamación o toxicidad (Davis et al., 2008; Jones, 2015). El comportamiento dominante de la especie puede mantenerlo en estrés constante y determinar sus niveles en la relación H:L (Valdebenito et al., 2021). Las diferencias entre los cuidados, alimentación, desparasitación de cada especie pueden modificar sus parámetros hematológicos (Campbell, 2015; Lashev et al., 2015).

\section{Hematocrito}

Esta variable refleja el volumen que ocupan los glóbulos rojos referente a la sangre entera (Galvez et al., 2009). De acuerdo a Lashev et al. (2015) su cantidad se relaciona directamente con el conteo de eritrocitos y hemoglobina, pero se ha Abdi-Hachesoo et al. (2011) reportaron diferencia entre hembras de la misma especie asociado a la altura de residencia. Okeudo et al. (2003) observaron menor Hto en hembras y machos de pato azteca respecto a nuestro estudio. Moreira et al. (2009) describen menor Hto en hembras y machos de guajolote que en el presente estudio. Nuestros parámetros para esta variable corresponden a los establecidos por Campbell (2015) para pollo de engorda, guajolote y pato doméstico. Así mismo nuestro porcentaje observado en machos de ave deportiva es similar al grupo control de Pineda-Leyva et al. (2015), también Oloyemi y Arewolo (2009) señalan una cantidad similar de esta variable en pato doméstico en temporada que no llueve. Es posible que el porcentaje de Hto puede ser diferente entre las especies debido sus hábitos de consumo de agua (Oloyemi y Arewolo, 2009), la masa corporal, adaptación a las condiciones ambientales y su tasa metabólica (McKechnie, 2007).

\section{Hemoglobina}

La hemoglobina es una proteína que transporta oxígeno hacia los tejidos. Okeudo et al. (2003) en pato azteca observaron menor concentración respecto a nuestros resultados. Por otro lado, nuestros parámetros para pollo de engorda, pato doméstico y guajolote están dentro de lo establecido por Campbell (2015). Moreira et al. (2009) reportan en guajolote una concentración de $\mathrm{Hb}$ mayor comparada a este estudio, de la misma forma Pineda-Leyva et al. (2015) señalan mayor concentración de $\mathrm{Hb}$ en machos de combate. La diferencia mencionada radicar en que el hto responde a la aclimatación por la diferencia de la altura sobre el nivel del mar y al nivel de testosterona presente en las diferentes especies de aves que responde a la eritropoyesis (Gonzales, 2011). La hemoglobina puede variar según la disponibilidad de hierro en la dieta y la capacidad intestinal para su absorción (Tako et al., 2010).

\section{CONCLUSIÓN}

Las variables hematológicas en aves deportivas, ganso común, pato doméstico, pato azteca, guajolote y pollo de engorda fueron similares para hembras y machos de la misma especie. Pero mostraron diferencias en las células blancas entre las especies estudiadas. Por los hallazgos encontrados es necesario seguir estudiando las células sanguíneas entre las especies debido a la localidad, la alimentación y el dimorfismo sexual.

\section{AGRADECIMIENTOS}

Los autores agradecen el apoyo recibido del Zoológico de Irapuato, Granja Mogotes, Granja San José de la Chiripa, Granja avícola de la Universidad de Guanajuato y a todos los particulares que colaboraron para realizar la investigación. 


\section{LITERATURA CITADA}

ABDI-HACHESOO B, Talebi A, Asri-Rezaei S. 2011. Comparative study on blood profiles of indigenous and Ross-308 broiler breeders. Global veterinaria. 7(3): 238-241. http://dx.doi.org/10.13140/RG.2.2.34589.97768

AENGWANICH W, Tanomtong A. 2007. Blood cell characteristics and hematological values of free ranging-red jungle fowl (gallus gallus) in Northeastern, Thailand. Journal of biological sciences. 7 (4): 689-692. https://doi.org/10.3923/jbs.2007.689.692

AGUSTÍ S. 2015. Estudio de la hematología y la bioquímica sanguínea de las rapaces nocturnas ibéricas. Tesis doctoral. Universidad Autónoma de Barcelona. 22-46. https://ddd.uab.cat/pub/tesis/2015/hdl_10803_329287/sam1de1.pdf

ALBOKHADAIM I. 2012. Hematological and some biochemical values of indigenous chickens in Al-Ahsa, Saudi Arabia during summer season. Asian journal of poultry science. 6 (4): 138145. https://doi.org/10.3923/ajpsaj.2012.138.145

AZEEZ O, Oloyemi F, Olanrewaju J. 2011. Age and sex influences on the haematology an erithrocyte osmotic fragility of the nigerian turkey. Research journal of veterinary sciences. 4(2): 43-49. https://doi.org/10.3923/rjvs.2011.43.49

BÍLKOVÁ B, Bainová Z, Zita L, Vinkler M. 2017. Different breeds, different blood: Cytometric analysis of whole blood celular composition in chicken breeds. Veterinary inmunology and immunopathology. 188: 71-77. https://doi.org/10.1016/j.vetimm.2017.05.001

CAMPBELL T. 2013. Chapter 9: Hematology. En Ritchie B, Harrison G, Harrison L. Avian medicine principles and application. 177-198. http://avianmedicine.net/wpcontent/uploads/2013/03/9.pdf

CAMPBELL T. 2015. Chapter 2: Peripheral blood of birds. En Campbell T. Exotic animal hematology. 37-66. https://doi.org/10.1002/9781118993705.ch2

CUCA-GARCÍA J, Gutiérrez-Arenas D, López- Pérez E. 2015. Avicultura de traspatio en México: Historia y caracterización. Agro productividad. 8 (4): 30-37. https://www.researchgate.net/publication/301553622_La_avicultura_de_traspatio_en_Mexico _Historia_y_Caracterizacion

DAVIS A, Maney D, Maerz J. 2008. The use of leukocyte profiles to mesure stress in vertebrates: a review for ecologist. Functional ecology. 22 (5): 760-772. https://doi.org/10.1111/j.1365-2435.2008.01467.x

FAIRBROTHER A, O'Loughlin D. 1990. Differential White blood cell values of the mallard (Anas platiyrhynchos) across different ages and reproductive states. Journal of wildlife disease. 26 (1): 78-82. https://doi.org/10.7589/0090-3558-26.1.78

FOO Y, Nakagawa S, Rhodes G, Simmons L. 2017. The effects of sex hormones on immune function: a meta-analisis. Biological reviews. 92(1): 551-571. https://doi.org/10.1111/brv.12243 
GÁLVEZ C, Ramírez G, Henry J. 2009. El laboratorio clínico en hematología de aves exóticas. Biosalud. 8: 178-188. http://www.scielo.org.co/pdf/biosa/v8n1/v8n1a20.pdf

GARBUS S, Chistensen J, Buchmann K, Jessen T, Lyngs P, Jacobsen M, Garbus G, Lund E, Garbus P, Madsen J, Thorup J, Sonne C. 2019. Hematology, blood biochemistry, parasites and pathology of common eider (Somateria mollisima) males during mortality event in the Baltic. Science of Total Enviroment. 683: 559-567. https://doi.org/10.1016/j.scitotenv.2019.05.281

GONZALES G. 2011. Hemoglobina y testosterona: Importancia en la aclimatación y adaptación a la altura. Revista peruana experimental y salud pública. 28(1): 92-100. https://doi.org/10.1590/S1726-46342011000100015

INSTITUTO NACIONAL DE ESTADÍSTICA Y GEOGRAFÍA (INEGI). 2017. Aspectos geográficos. Anuario estadístico de Guanajuato.

http://internet.contenidos.inegi.org.mx/contenidos/Productos/prod_serv/contenidos/espanol/bv inegi/productos/nueva_estruc/anuarios_2017/702825092146.pdf

JONES M. 2015. Avian hematology. Veternary clinics exotic animals. 18(1): 51-61. https://doi.org/10.1016/j.cvex.2014.09.012

LASHEV L, Atanasova S, Dinev T. 2015. Interspecies and gender-related variations of some haematological parameters in galliformes birds species. Bulgarian journal of veterinary medicine. 18 (4): 325-337. https://doi.org/10.15547/bjvm.783

MARTÍNEZ-SILVESTRE A, Lavín S, Cuenca R. 2011. Hematología y citología sanguínea en reptiles. Clínica veterinaria de pequeños animales. 31(3): 131-141. https://ddd.uab.cat/record/128943

MARTINHO F. 2009. Indications and techniques for blood transfusion in birds. Journal of exotic pet medicine. 18(2): 112-116. https://doi.org/10.1053/j.jepm.2009.04.001

MCKECHNIE A. 2007. Phenotypic flexibility in basal metabolic rate and the changing view of avian physiological diversity: a review. Journal of comparative physiology B. 178: 235-247. https://doi.org/10.1007/s00360-007-0218-8

MITCHELL E, Johns J. 2008. Avian hematology and related disorders. Veterinary clinics exotic animal practice. 11(3): 501-522. https://doi.org/10.1016/j.cvex.2008.03.004

MONTALVO C. 2017. Reacción inflamatoria en tejido sanguíneo y hematopoyesis. Departamento de biología celular y tisular UNAM. http://www.facmed.unam.mx/deptos/biocetis/PDF/Portal de Recursos en Linea/Apuntes/Tejido-sanguineo.pdf

MOREIRA E, Paulillo A, Viera G, Lapera I, Pereira A, Junior L, Denadai J, Jurandir F. 2009. Hematology of the bronze turkey (Meleagris gallopavo) variations with age and gender. Journal of poultry science. 8(8): 752-754. https://doi.org/10.3923/ijps.2009.752.754 
OKEUDO N, Okoli I, Igwe G. 2003. Hematological characteristics of ducks (Cairina moschata) of southeastern Nigeria. Tropicultura. 21(2): 61-65.

https://www.researchgate.net/publication/45266353_Hematological_Characteristics_of_Ducks Cairina_moschata_of_Southeastern_Nigeria

OLOYEMI F, Arowolo R. 2009. Seasonal variations in the haematological values of the nigerian duck (Anas platyrhynchos). Journal of poultry science. 8(8): 813-815. https://doi.org/10.3923/ijps.2009.813.815

PINEDA-LEYVA E, Talavera-Rojas M, Peña-Romero A, Soriano-Vargas E, Alejandri-Cortes C. 2015. Perfiles hematológicos en respuesta a la administración de inmunomoduladores inespecíficos en aves de combate (Gallus gallus gallus). Revista científica. 8(5): 368-374. http://ri.uaemex.mx/handle/20.500.11799/40015

PISTONE J, Heatley J, Campbell T, Voelker G. 2017. Assessing Paseriformes health in south Texas via select venous analytes. Journal of comparative biochemistry and physiology. 210B: 64-71. https://doi.org/10.1016/j.cbpb.2017.06.002

SAMOUR J, 2007. Chapter 22: Diagnostic value of hematology. En Harrison G, Ligthfoot T. Clinical Avian Medicine. 2: 588-610. http://avianmedicine.net/wpcontent/uploads/2013/08/22_hematology.pdf

SCANES C. 2015. Blood. Sturkie's avian physiology. 6ta edición. 167-191. https://doi.org/10.1016/B978-0-12-407160-5.00010-5

TAKO E, Rutze M, Glahn. 2010. Using the domestic chicken (Gallus gallus) as an in vivo model for iron bioavailability. Poultry science. 89(3): 514-521. https://doi.org/10.3382/ps.2009-00326

VALDEBENITO J, Halimubieke N, Lendvai A, Figuerola J, Eichhorn G, Székely T. 2021. Seasonal variation in sex-specific immunity in wild birds. Scientific reports. 11(1): 1-11. https://doi.org/10.1038/s41598-020-80030-9 